\title{
Theoretical Back Analysis of Internal Forces of Primary Support in Deep Tunnels
}

\author{
He Wenzheng ${ }^{1,2}$, Xu Linsheng ${ }^{1, *}$ and Wang Lili ${ }^{3}$ \\ ${ }^{l}$ Chongqing Municipal Key Laboratory of Mountain Highway \& Waterway Transportation Geological Disaster Reduction, Chongqing \\ Jiaotong University, Chongqing 400074, China \\ ${ }^{2}$ School of Urban Construction Engineering, Chongqing Radio \& Television University, Chongqing 401520, China \\ ${ }^{3}$ School of Management, Cardiff Metropolitan University, Cardiff, CF52YB, Wales
}

Received 20 September 2018; Accepted 5 January 2019

\begin{abstract}
The internal forces of primary support obtained by theoretical analysis and numerical simulation often differ significantly from actual data due to the uncertainty of parameters of the surrounding rock and the field construction conditions in tunnels. This study proposed a novel back-analysis method to accurately evaluate the internal forces of the primary support on the basis of radial displacement and contact stress. According to the geometric characteristics and mechanical properties of the primary support in deep tunnels, a mechanical model was established based on theory of circular curved beam. The analytical solutions of these internal forces were then deduced. A case study was conducted to analyze the internal forces of the primary support obtained using the proposed method. Results demonstrate that the internal forces of the primary support of symmetrically loaded tunnels can be directly evaluated by radial displacement and contact stress. The peak-values of internal forces appear in vault, arch springing and the section with a $\theta$ angle of $60^{\circ}$. This study provides reference for the dynamic design and optimization of construction for the primary support in tunnels.
\end{abstract}

Keywords: Primary support, Radial displacement, Contact stress, Back analysis

\section{Introduction}

Composite lining systems are extensively used in tunnel engineering. According to the load-carrying mechanism of the composite lining, the primary support is a major loadcarrying structure. Studying the internal forces of the primary support of tunnels is important to provide reference for analyzing safety in tunnel construction and basis for feedback evaluation of the rationality of the tunnel design. However, the internal forces of primary supports obtained by theoretical analysis and numerical simulation often differ significantly from the actual data due to the uncertainty of the parameters of the surrounding rock and the field construction conditions in tunnels. Therefore, the calculated results cannot provide reliable criteria for tunnel design and construction [1]. Evaluating the stress states of the structures and the stability of surrounding rocks by using monitoring data has become an important method for ensuring safety in tunnel construction [2-6]. In this regard, quantitative and objective back analyses of the stress conditions of the surrounding rocks and the supporting structures should be conducted based on monitoring data.

Numerous studies have reported on back analysis for tunnel engineering based on monitoring and measurement data [7-10]. However, existing literature mainly focuses on the back analysis of the mechanical parameters of surrounding rocks and lacks efficient direct calculation methods for the internal forces of supporting structures. Moreover, differences exist between mechanical

\footnotetext{
*E-mail address: phoniex8210@sina.com

ISSN: 1791-2377 С 2019 Eastern Macedonia and Thrace Institute of Technology. All rights reserved. doi:10.25103/jestr.121.03
}

assumptions of the primary support and actual working conditions. Therefore, accurately calculating the internal forces of the primary support by using monitoring data remains a problem and demands prompt solutions.

This study extensively analyzed the mechanics of primary support in tunnels. The analytic expressions for the internal forces of the primary support in the detachment and in the elastic resistance zones were deduced from the theory of circular curved beam. The distribution characteristics of the internal forces were analyzed to quickly and accurately evaluate the stress states of the primary support and provide references for the dynamic design and optimization of construction in tunnel engineering.

\section{State of the art}

Numerous works have been conducted on monitoring measurements and back analysis of underground structures. Moreira et al. [11] developed an evolution strategy (ES) in the back analysis of geomechanical parameters in underground structures based on an evolutionary computation approach. ES showed satisfactory performance in terms of robustness and efficiency in most of the tested synthetic problem cases even for highly complex error functions. However, this method did not thoroughly analyze the stress characteristics of the support structures. Dehghan et al. [12] proposed an economic and time-saving univariate back-analysis method based on convergence data; however, the gained parameters were higher than those in the soil mechanical test. Zhang et al. [13] discussed the recognition of linear elastic transverse isotropy rock parameters, the optimized layout of measuring points, and the optimization 
algorithm by analytic method; this analytical method was only applicable to round tunnels. Deng et al. [14] constructed a parameter recognition method based on a fluid-solid coupling model by combining the finite element method and the adaptive genetic algorithm. However, the stress condition of the support structure has not been thoroughly studied. Zhu et al. [15] proposed a displacement back-analysis method based on the artificial bee colony algorithm and the least square method. However, this method was time-consuming for problems without analytical solutions. Miranda et al. [16-17] proposed an inverse algorithm of the mechanical parameters of rock mass by combining the optimization algorithm and a 3D model, which proposed high requirements on observation data. Yazdani et al. [18] constructed the back-analysis method based on continuous and intermittent numerical simulations of the displacement to recognize geomechanical properties, stress ratios, and the joint parameters of rocks. Based on deformation analysis data, Kodama et al. [19] proposed a back-analysis method, which estimated regional geostress, and Young's modulus, determining that back analysis based on the changing distances of measuring points was more reliable than back analysis based on direct relative displacement. Janin et al. [20-21] simulated the complicated $3 \mathrm{D}$ effect involving the interaction of the excavation process, the reinforcement, the tunnel-supporting load, and the foundation counterforce utilizing the $3 \mathrm{D}$ numerical inversion method. Sun et al. [22] proposed a dynamic back-prediction model to predict the deformations and the failure modes of surrounding rocks in tunnels and to evaluate the stability of surrounding rocks during construction.. All the aforementioned studies focused on the back analysis of the mechanical parameters of the surrounding rock. The theoretical back-analysis methods based on theory of beam on elastic foundation provided a new approach for evaluating the mechanical properties of tunnel support. Based on contact and steel arch stresses, Wen et al. [23-24] deduced the analytic formula for the back analysis of the internal force of the primary support in tunnels utilizing theory of circular curved beam on elastic foundation. Wang et al. [25] presented the inversion-analysis method of the internal force of the primary support in the horseshoe-shaped tunnel. Cheng et al. [26] proposed an inversion calculation method of the internal forces of support structures in multiarc tunnels. However, the three aforementioned theoretical back-analysis methods were all based on theory of beam on elastic foundation, which is not applicable for tunnel support.

The results of the aforementioned research were mainly gained from the back analysis of the mechanical parameters of rocks. However, limited studies have been conducted directly on the back analysis of the stress characteristics of the primary support. The limited back-analysis studies concerning the mechanical properties of the primary support based on analytical methods all hypothesize that the primary support is a beam on elastic foundation. This hypothesis differs significantly with the actual stress state of a tunnel. The calculation method derived by the hypothesis reveals considerable errors. In the present study, the stress characteristics of the primary support of the tunnel were analyzed extensively. The expressions of the internal forces of the primary support in detachment and elastic resistance zones were deduced from theory of circular curved beam. Moreover, the internal force distribution of the primary support and its time-variant characteristics were discussed. The proposed theoretical method considered the loaddistribution characteristic of primary support. The research conclusions conformed to the actual stress of the tunnel and provided references for the optimization of the primary support design and construction in tunnels.

The remainder of this study is organized as follows: Section 3 establishes the mechanical model and proposes the back-analysis method for the internal force of the primary support in deep tunnels; Section 4 discusses the applicability of the method through case studies and Section 5 summarizes the conclusions.

\section{Methodology}

3.1 Shortcomings of existing back-analysis theories based on elastic foundation beam method

In existing back-analysis methods based on theory of beam on elastic foundation [23-26], the contact stresses between the shotcrete and the surrounding rocks were viewed as the elastic resistance of the foundation. However, this assumption is not accurate because the primary support bears active and passive loads (elastic resistance of surrounding rocks), and the contact stress between the shotcrete and the surrounding rocks may be caused by active loads rather than solely by the elastic resistance of the surrounding rocks. At positions near the vault, the shotcrete deforms toward the tunnel under the active loads, thus forming the detachment zone. Under this circumstance, the primary support only bears active loads and no elastic resistance of the surrounding rocks. At the positions where the structure deforms toward the surrounding rock, the surrounding rocks produce a passive elastic resistance to the primary support, forming the resistance zone. The deformation curve of the surrounding rocks is presented in Fig.1. In this case, the primary support in the resistance zone bears active loads and the elastic resistance of the surrounding rocks. In fact, the primary support in a tunnel in benching construction develops free deformation, and the surrounding rocks produce no elastic resistance after the excavation of the upper benching and before the excavation of the surrounding rocks in the lower benching. Therefore, the internal forces of the primary support cannot be calculated according to theory of beam on elastic foundation, and the calculation model should be reconstructed according to the mechanical mechanism of the support structure.

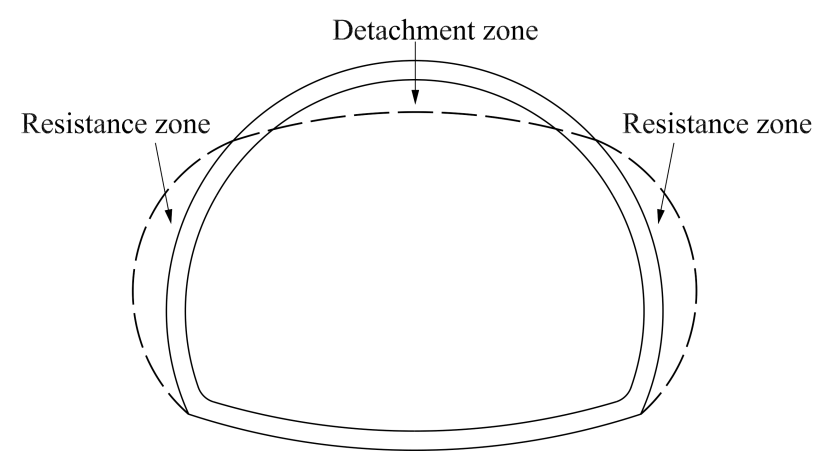

Fig. 1. Deformation curve of surrounding rocks

3.2 The improved governing differential equation for primary support according to shortcomings

The section forms of highway tunnels are generally multicentered circle. The primary support is viewed as a circular curved beam, and the geometric shape is presented in Fig. 2. 
The inner and outer radius of the circular curved beam are $r_{0}$ and $r_{1}$, respectively, and the central radius of the section is $r=\left(r_{0}+r_{1}\right) / 2$. To construct the differential equations of the entire circular curved beam range, the $\delta(\omega)$ function is introduced. Let

$\delta(\omega)= \begin{cases}1 & \omega<0 \\ 0 & \omega \geq 0\end{cases}$

where $\omega$ is the radial displacement. The radial displacement pointing to the center of a circle is positive.

Micro unit $r d \theta$ is selected. According to the stress condition (Fig. 3), the equilibrium equations of force and the bending moment are established

$$
\begin{aligned}
& d Q(\theta)+N(\theta) d \theta+[K \delta(\omega) \omega(\theta) \\
& +p(\theta)] r_{1} d \theta=0 \\
& Q(\theta) d \theta-d N(\theta)=0 \\
& d M(\theta)-r d N(\theta)=0
\end{aligned}
$$

where $\omega(\theta)$ is the radial displacement, $K$ is the coefficient of the elastic resistance of surrounding rocks, $A$ is the sectional area, $I$ is the moment of inertia of the section, $p(\theta)$ is the radial force produced by the active loads, $M(\theta)$ is the bending moment, $Q(\theta)$ is the shear force, and $N(\theta)$ is the axial force. The directions of all the forces in Fig. 3 are positive.

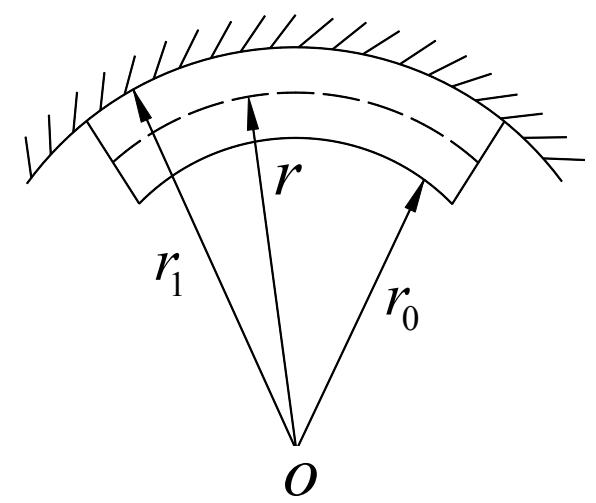

Fig. 2. Geometric appearance of primary support

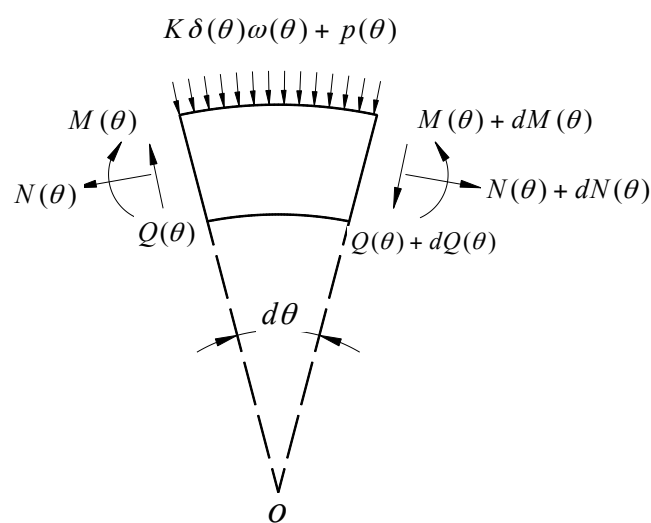

Fig. 3. Stress sketch of micro unit
According to the mechanics of the structure, there is a relationship between the radial displacement $(\omega(\theta))$ and the internal forces of the circular curved beam.

$\frac{d^{2} \omega(\theta)}{d \theta^{2}}+\omega(\theta)=\frac{M(\theta) r^{2}}{E_{c} I}+\frac{N(\theta) r}{E_{c} A}$

where $E_{c}$ is the elasticity modulus of the primary support. By utilizing the steel arch and the shotcrete combined support, $E_{c}$ is the equivalent elasticity modulus.

After the first and third derivations of $\theta$ in Eq. (4), the following equations can be obtained:

$$
\begin{aligned}
& \frac{d^{3} \omega(\theta)}{d \theta^{3}}+\frac{d \omega(\theta)}{d \theta}=\frac{r^{2} d M(\theta)}{E_{c} I d \theta}+\frac{r d N(\theta)}{E_{c} A d \theta} \\
& \frac{d^{5} \omega(\theta)}{d \theta^{5}}+\frac{d^{3} \omega(\theta)}{d \theta^{3}}=\frac{r^{2} d^{3} M(\theta)}{E_{c} I d \theta^{3}}+\frac{r d^{3} N(\theta)}{E_{c} A d \theta^{3}}
\end{aligned}
$$

Substituting Eq. (5) into Eq. (6), the following equation can be obtained:

$\frac{d^{5} \omega(\theta)}{d \theta^{5}}+2 \frac{d^{3} \omega(\theta)}{d \theta^{3}}+\frac{d \omega(\theta)}{d \theta}=\frac{r^{2} d^{3} M(\theta)}{E_{c} I d \theta^{3}}+\frac{r d^{3} N(\theta)}{E_{c} A d \theta^{3}}$

The following five-order ordinary radial displacement differential equation can be derived by substituting Eqs. (1)(3) into Eq. (7):

$$
\frac{d^{5} \omega(\theta)}{d \theta^{5}}+2 \frac{d^{3} \omega(\theta)}{d \theta^{3}}+m^{2} \frac{d \omega(\theta)}{d \theta}=F \frac{d p(\theta)}{d \theta}
$$

where $m^{2}=1-K F \delta(\omega)$ and $F=-\frac{\left(r^{3} A+r I\right) r_{1}}{E_{c} I A}$.

In the deep tunnel, the radial component of the surrounding rock pressure can be expressed as follows:

$$
p(\theta)=\bar{p} \cos (\theta)+\lambda \bar{p} \sin (\theta)
$$

where $\bar{p}$ is the vertical component of active loads and $\lambda$ is the lateral pressure coefficient. The section where the vertical axis of the tunnel lies is utilized as the initial section. For this section, it is regulated that $\theta=0$. The clockwise direction is positive and the counterclockwise direction is negative. The sign of $\lambda$ is the same as that of $\theta \cdot \bar{p}$, and $\lambda$ can be calculated from the measurement data.

The following governing differential equation can be derived by substituting Eq. (9) into Eq. (8):

$$
\begin{aligned}
& \frac{d^{5} \omega(\theta)}{d \theta^{5}}+2 \frac{d^{3} \omega(\theta)}{d \theta^{3}}+m^{2} \frac{d \omega(\theta)}{d \theta}= \\
& F(-\bar{p} \sin \theta+\lambda \bar{p} \cos \theta)
\end{aligned}
$$

In the detachment zone, the elastic resistance of the surrounding rocks is neglected. Thus, $\delta(\omega)=0$, and Eq. (10) can be expressed as follows: 
$\frac{d^{5} \omega(\theta)}{d \theta^{5}}+2 \frac{d^{3} \omega(\theta)}{d \theta^{3}}+\frac{d \omega(\theta)}{d \theta}=$

$F(-\bar{p} \sin \theta+\lambda \bar{p} \cos \theta)$

The governing differential equation can be solved in two different cases.

3.2.1 Solving the governing differential equation with considerations to elastic resistance

In the resistance zone, $\delta(\omega)=1$ and $m^{2}$ is greater than 1 .

The governing equation of the circular curved beam is a five-order ordinary nonhomogeneous differential equation (Eq. [10]). The complete solution $(\omega(\theta))$ can be expressed as the sum of the general solution of the homogeneous differential equation $\left(\omega_{0}(\theta)\right)$ and the particular solution of the nonhomogeneous differential equation $\left(\omega^{*}(\theta)\right)$, as follows:

$\omega(\theta)=\omega_{0}(\theta)+\omega^{*}(\theta)$

According to theory of differential equations, the general solution of the homogeneous equation $\left(\omega_{0}(\theta)\right)$ can be expressed as follows:

$\omega_{0}(\theta)=C_{1}+C_{2} \operatorname{ch} \alpha \theta \cos \beta \theta+$

$C_{3} \operatorname{sh} \alpha \theta \cos \beta \theta+C_{4} \operatorname{ch} \alpha \theta \sin \beta \theta+C_{5} \operatorname{sh} \alpha \theta \sin \beta \theta$

where $\alpha=\sqrt{\frac{m-1}{2}}, \beta=\sqrt{\frac{m+1}{2}}$, and $C_{1}-C_{5}$ are integration constants. The particular solution of the nonhomogeneous differential equation can be calculated by the undetermined coefficient method:

$\omega^{*}(\theta)=\frac{F \bar{p}}{m^{2}-1} \cos \theta+\frac{\lambda F \bar{p}}{m^{2}-1} \sin \theta$

Substituting Eqs. (13)-(14) into Eq. (12), the following complete solution of the governing differential equation can be obtained:

$\omega(\theta)=C_{1}+C_{2} \operatorname{ch} \alpha \theta \cos \beta \theta+C_{3} \operatorname{sh} \alpha \theta \cos \beta \theta$

$+C_{4} \operatorname{ch} \alpha \theta \sin \beta \theta+C_{5} \operatorname{sh} \alpha \theta \sin \beta \theta$

$+\frac{F \bar{p}}{m^{2}-1} \cos \theta+\frac{\lambda F \bar{p}}{m^{2}-1} \sin \theta$

For a symmetrically loaded tunnel, $\omega(-\theta)=\omega(\theta)$ due to the symmetry of geometry and loads. Substituting $\omega(-\theta)=\omega(\theta)$ into Eq. (15), $C_{3}=C_{4}=0$ can be derived.

Therefore, Eq. (15) can be simplified as follows:

$\omega(\theta)=C_{1}+C_{2} \operatorname{ch} \alpha \theta \cos \beta \theta+$

$C_{3} \operatorname{sh} \alpha \theta \sin \beta \theta+\frac{F \bar{p}}{m^{2}-1} \cos \theta+\frac{\lambda F \bar{p}}{m^{2}-1} \sin \theta$

3.2.2 Solving the governing differential equation without consideration to elastic resistance

The primary support in the detachment zone only bears active loads. In this case, $\delta(\omega)=0$, thus $m^{2}=1$. The general solution of governing equation (Eq. [11]) can be expressed as follows:

$\omega_{0}(\theta)=C_{1}+C_{2} \sin \theta+C_{3} \cos \theta+C_{4} \theta \sin \theta+C_{5} \theta \cos \theta$

The particular solution of the nonhomogeneous differential equation can be gained by utilizing the constant variation method.

$$
\begin{aligned}
& \omega^{*}(\theta)=\frac{F \bar{p}}{8}\left(6 \cos \theta+4 \theta \sin \theta-\theta^{2} \cos \theta\right) \\
& +\frac{\lambda F \bar{p}}{8}\left(6 \sin \theta-4 \theta \cos \theta-\theta^{2} \sin \theta\right)
\end{aligned}
$$

The following complete solution can be derived by substituting Eqs. (17)-(18) into Eq. (12):

$\omega(\theta)=C_{1}+C_{2} \sin \theta+C_{3} \cos \theta+C_{4} \theta \sin \theta+$

$C_{5} \theta \cos \theta+\frac{F \bar{p}}{8}\left(6 \cos \theta+4 \theta \sin \theta-\theta^{2} \cos \theta\right)$

$+\frac{\lambda F \bar{p}}{8}\left(6 \sin \theta-4 \theta \cos \theta-\theta^{2} \sin \theta\right)$

Similarly, according to the symmetry, the Eq. (19) can be simplified as follows:

$$
\begin{aligned}
& \omega(\theta)=C_{1}+C_{2} \cos \theta+C_{3} \theta \sin \theta+ \\
& \frac{F \bar{p}}{8}\left(6 \cos \theta+4 \theta \sin \theta-\theta^{2} \cos \theta\right)+ \\
& \frac{\lambda F \bar{p}}{8}\left(6 \sin \theta-4 \theta \cos \theta-\theta^{2} \sin \theta\right)
\end{aligned}
$$

\subsection{Internal force analysis of primary support}

The following expression of the shear force can be derived by substituting Eqs. (2)-(3) into Eq. (5):

$$
Q(\theta)=\left(\frac{r^{3}}{E_{c} I}+\frac{r}{E_{c} A}\right)^{-1}\left[\frac{d^{3} \omega(\theta)}{d \theta^{3}}+\frac{d \omega(\theta)}{d \theta}\right]
$$

The following expression of the axial force can be obtained by substituting Eq. (1) into Eq. (21):

$$
\begin{aligned}
& N(\theta)=-\left(\frac{r^{3}}{E_{c} I}+\frac{r}{E_{c} A}\right)^{-1}\left[\frac{d^{4} \omega(\theta)}{d \theta^{4}}+\frac{d^{2} \omega(\theta)}{d \theta^{2}}\right] \\
& -K r_{1} \delta(\omega) \omega(\theta)-\bar{p} r_{1} \cos \theta-\lambda \bar{p} r_{1} \sin \theta
\end{aligned}
$$

The expression of the bending moment can be expressed by substituting Eq. (4) into Eq. (22), as follows:

$$
\begin{aligned}
& M(\theta)=\frac{E_{c} I}{r^{2}}\left\{\frac{d^{2} \omega(\theta)}{d \theta^{2}}+\left(\frac{r}{E_{c} A} K \delta(\omega) r_{1}+1\right) \omega(\theta)+\right. \\
& \left(\frac{A r^{2}}{I}+1\right)^{-1}\left[\frac{d^{4} \omega(\theta)}{d \theta^{4}}+\frac{d^{2} \omega(\theta)}{d \theta^{2}}\right]+
\end{aligned}
$$

$\left.\frac{r}{E_{c} A}\left(\bar{p} r_{1} \cos \theta+\lambda \bar{p} r_{1} \sin \theta\right)\right\}$ 


\subsubsection{Internal force analysis of primary support in the} resistance zone

In the resistance zone, the expressions of the internal forces can be derived by substituting Eq. (16) into Eqs. (21)-(23):

$$
\begin{aligned}
& Q(\theta)=T\left[\left(C_{2} A_{1}-C_{3} A_{2}\right) \operatorname{sh} \alpha \theta \cos \beta \theta+\right. \\
& \left.\left(C_{2} A_{2}+C_{3} A_{1}\right) \operatorname{ch} \alpha \theta \sin \beta \theta\right]
\end{aligned}
$$

where $A_{1}=\alpha^{3}-3 \alpha \beta^{2}+\alpha, \quad A_{2}=\beta^{3}-3 \alpha^{2} \beta-\beta, \quad$ and $T=\left(\frac{r_{i}^{3}}{E_{c} I}+\frac{r_{i}}{E_{c} A}\right)^{-1}$.

$N(\theta)=\left[\left(-T C_{2} B_{1}+T C_{3} B_{2}-K r_{1} \delta(\omega) C_{2}\right) \operatorname{ch} \alpha \theta \cos \beta \theta+\right.$

$\left.\left(-T C_{2} B_{2}-T C_{3} B_{1}-K r_{1} \delta(\omega) C_{3}\right) \operatorname{sh} \alpha \theta \sin \beta \theta\right]-$

$K r_{1} \delta(\omega) \frac{F \bar{p}}{m^{2}-1} \cos \theta-K r_{1} \delta(\omega) \frac{\lambda F \bar{p}}{m^{2}-1} \sin \theta-$

$\bar{p} r_{1} \cos \theta-\lambda \bar{p} r_{1} \sin \theta$

where $\quad B_{1}=\alpha^{4}-6 \alpha^{2} \beta^{2}+\beta^{4}+\alpha^{2}-\beta^{2}$ and

$B_{2}=-4 \alpha^{3} \beta+4 \alpha \beta^{3}-2 \alpha \beta$.

$M(\theta)=\frac{E_{c} I}{r^{2}}\left\{C_{1} G-\right.$

$\left[\left(B_{1} C_{2} D-B_{2} C_{3} D+C_{2} E_{1}-C_{3} E_{2}+C_{2} G\right) \operatorname{ch} \alpha \theta \cos \beta \theta+\right.$

$\left.\left(B_{1} C_{3} D+B_{2} C_{2} D+C_{3} E_{1}+C_{2} E_{2}+C_{3} G\right) \operatorname{sh} \alpha \theta \sin \beta \theta\right]+$

$\left.\left(D F \bar{p}+\frac{\bar{p} r_{1} r}{E_{c} A}\right) \cos \theta+\lambda\left(D F \bar{p}+\frac{\bar{p} r_{1} r}{E_{c} A}\right) \sin \theta\right\}$

where

$D=\left(\frac{A r^{2}}{I}+1\right)^{-1}, G=\left(\frac{r}{E_{c} A} K r_{1}+1\right), E_{1}=\alpha^{2}-\beta^{2}$

and

$E_{2}=-2 \alpha \beta$.

\subsubsection{Internal force analysis of primary support in the} detachment zone

In the detachment zone, without consideration to the elastic resistance of the surrounding rocks, the expressions of the internal forces can be derived by substituting Eq. (20) into Eqs. (21)-(23):

$$
\begin{aligned}
& Q(\theta)=T\left[\left(-2 C_{3}-\frac{F \bar{p}}{4}\right) \sin \theta+\frac{\lambda F \bar{p}}{4} \cos \theta+\right. \\
& \left.\frac{\lambda F \bar{p}}{2} \theta \sin \theta+\frac{F \bar{p}}{2} \theta \cos \theta\right] \\
& N(\theta)=-T\left[\left(\frac{\lambda F \bar{p}}{4}+\frac{\lambda \bar{p} r_{1}}{T}\right) \sin \theta+\right. \\
& \left.\left(-2 C_{3}+\frac{F \bar{p}}{4}+\frac{\bar{p} r_{1}}{T}\right) \cos \theta-\frac{F \bar{p}}{2} \theta \sin \theta+\frac{\lambda F \bar{p}}{2} \theta \cos \theta\right]
\end{aligned}
$$

$$
\begin{aligned}
& M(\theta)=\frac{E_{c} I}{r^{2}}\left[C_{1}+\left(\frac{D \lambda F \bar{p}}{4}+\frac{3 \lambda F \bar{p}}{4}+\frac{\lambda \bar{p} r r_{1}}{E_{c} A}\right) \sin \theta+\right. \\
& \left(-2 C_{3} D+2 C_{3}+\frac{D F \bar{p}}{4}+\frac{3 F \bar{p}}{4}+\frac{\bar{p} r r_{1}}{E_{c} A}\right) \cos \theta+ \\
& \left.\left(-\frac{D F \bar{p}}{2}+\frac{F \bar{p}}{2}\right) \theta \sin \theta+\left(\frac{D \lambda F \bar{p}}{2}-\frac{\lambda F \bar{p}}{2}\right) \theta \cos \theta\right]
\end{aligned}
$$

\subsection{Solving integration constant by measurement data}

To solve the integration constants, the vertical component $\bar{p}$ and lateral pressure coefficient $\lambda$ should be first calculated by the measured contact stress between the surrounding rocks and the shotcrete. For a symmetrically loaded tunnel, $\bar{p}$ and $\lambda$ can be obtained by the stress data of two different measuring points. By combining the measured data of the radial displacement of the three measuring points on the section, the three unknown integration constants can be calculated. The layout of the measuring points is presented in Fig. 4.

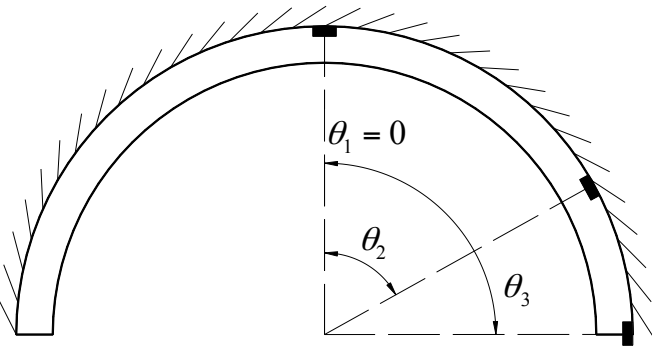

Fig. 4. Layout of the measuring points of the displacement and contact stress

The following equations are obtained by substituting the measured contact stress data into Eq. (9):

$$
\begin{aligned}
& p\left(\theta_{1}\right)=\bar{p} \cos \theta_{1}+\lambda \bar{p} \sin \theta_{1} \\
& p\left(\theta_{2}\right)=\bar{p} \cos \theta_{2}+\lambda \bar{p} \sin \theta_{2}
\end{aligned}
$$

The following expressions can be derived according to Eqs. (30) and (31)

$$
\begin{gathered}
\lambda=-\frac{p\left(\theta_{2}\right) \cos \theta_{1}-p\left(\theta_{1}\right) \cos \theta_{2}}{p\left(\theta_{2}\right) \sin \theta_{1}-p\left(\theta_{1}\right) \sin \theta_{2}} \\
\bar{p}=\frac{p\left(\theta_{2}\right) \sin \theta_{1}-p\left(\theta_{1}\right) \sin \theta_{2}}{\cos \theta_{2} \sin \theta_{1}-\cos \theta_{1} \sin \theta_{2}}
\end{gathered}
$$

Currently, the non-contact measurement method has been extensively utilized in monitoring tunnel measurements. This method can accomplish measurement tasks effectively and accurately. The total station instrument can be employed to measure horizontal and vertical displacement at the different section measuring points in tunnels. The following expressions can be derived utilizing the rotary formula of the coordinate system:

$\phi(\theta)=\Delta x \cos (\theta)+\Delta y \sin (\theta)$

$\omega(\theta)=-\Delta x \sin (\theta)+\Delta y \cos (\theta)$ 
where $\Delta x$ and $\Delta y$ are the horizontal and the vertical displacements gained by the total station instrument, $\omega(\theta)$ is the radial displacement, and $\phi(\theta)$ is the tangential displacement. The positive direction of the displacement is presented in Fig. 5.

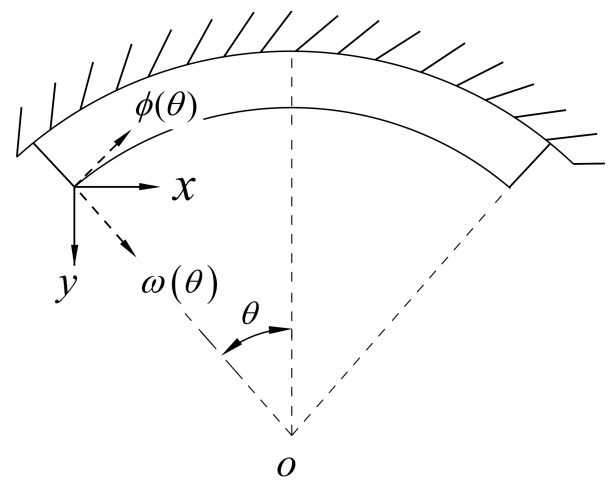

Fig. 5. Transformation of the coordinate system

The integration constants $C_{1}-C_{3}$ can be solved by radial displacement at different measuring points of the section. Three measuring points of the radial displacement are set on the symmetrically loaded tunnel (Fig. 4). The angles between these measuring points and the vertical axis of the tunnel are $\theta_{1}-\theta_{3}$. The clockwise direction is positive. The three-order linear equation set can be derived by substituting the measurement data into the expression of the radial displacement.

$$
\left[\begin{array}{lll}
1 & a_{1} & b_{1} \\
1 & a_{2} & b_{2} \\
1 & a_{3} & b_{3}
\end{array}\right]\left[\begin{array}{l}
C_{1} \\
C_{2} \\
C_{3}
\end{array}\right]=\left[\begin{array}{l}
J_{1} \\
J_{2} \\
J_{3}
\end{array}\right]
$$

In the resistance zone, the parameters of Eq. (36) can be expressed as follows:

$a_{i}=\operatorname{ch} \alpha \theta_{i} \cos \beta \theta_{i}, b_{i}=\operatorname{sh} \alpha \theta_{i} \sin \beta \theta_{i}$ and

$J_{i}=\omega\left(\theta_{i}\right)-\frac{F \bar{p}}{m^{2}-1} \cos \theta_{i}-\frac{\lambda F \bar{p}}{m^{2}-1} \sin \theta_{i}$.

In the detachment zone, the parameters of Eq. (36) can be expressed as follows:

$a_{i}=\cos \theta_{i}, b_{i}=\theta_{i} \sin \theta_{i}$ and

$J_{i}=\omega\left(\theta_{i}\right)-\frac{F \bar{p}}{8}\left(6 \cos \theta_{i}+4 \theta_{i} \sin \theta_{i}-\theta_{i}^{2} \cos \theta_{i}\right)-$

$\frac{\lambda F \bar{p}}{8}\left(6 \sin \theta_{i}-4 \theta_{i} \cos \theta_{i}-\theta_{i}^{2} \sin \theta_{i}\right)$

Substitute the measured radial displacement data into Eq. (36), and the following expressions can be derived:

$$
\left[\begin{array}{l}
C_{1} \\
C_{2} \\
C_{3}
\end{array}\right]=\left[\begin{array}{lll}
1 & a_{1} & b_{1} \\
1 & a_{2} & b_{2} \\
1 & a_{3} & b_{3}
\end{array}\right]^{-1}\left[\begin{array}{l}
J_{1} \\
J_{2} \\
J_{3}
\end{array}\right]
$$

The internal forces of the primary structure can be obtained by substituting the integration constants $C_{1}-C_{3}$ into Eqs. (24)-(26).

\section{Analysis Results and Discussion}

\subsection{Monitoring data of the Lvjialiang tunnel}

The Lujialiang tunnel (Fig. 6) is located in Chadiping, Zhong County, Chongqing City, China. This section of the tunnel is three-centered circle with an arch radius of $5.5 \mathrm{~m}$. The tunnel was excavated by step method. The central angle of the upper bench is $\pi / 2$. The primary support with the longitudinal unit length is taken as the calculation object. The geometric parameters of the upper benching section of the primary support are listed in Table 1. The material parameters are listed in Table 2.

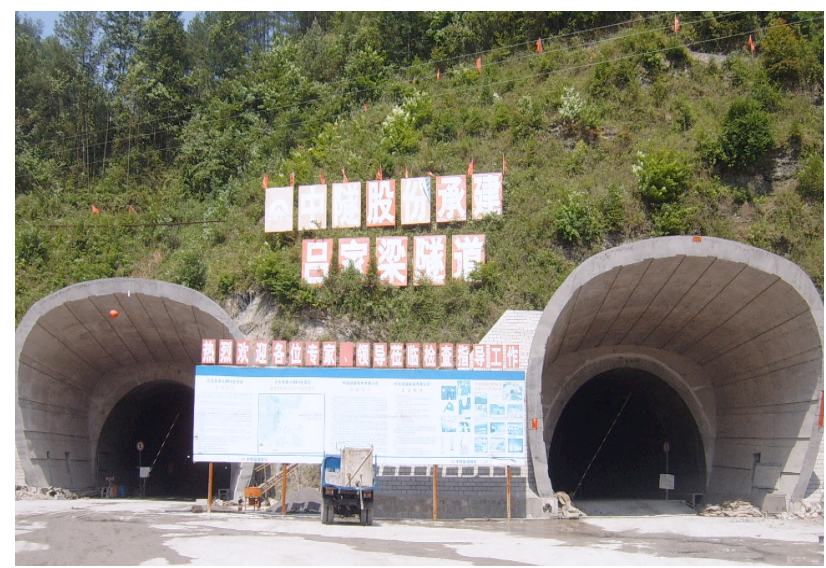

Fig. 6. Photo of the Lujialiang tunnel

Table 1. Geometric parameters of the upper bench section

\begin{tabular}{c|c|c|c|c}
\hline $\begin{array}{c}\text { Thickness of } \\
\text { the shotcrete } \\
h(m)\end{array}$ & $\begin{array}{c}\text { Excavation } \\
\text { radius } \\
r_{1}(m)\end{array}$ & $\begin{array}{c}\text { Inner } \\
\text { radius } \\
r_{0}(m)\end{array}$ & $\begin{array}{c}\text { Sectional } \\
\text { area } \\
A\left(\mathrm{~m}^{2}\right)\end{array}$ & $\begin{array}{c}\text { Central } \\
\text { angle } \\
\theta(\mathrm{rad})\end{array}$ \\
\hline 0.20 & 5.60 & 5.40 & 0.2 & $\pi / 2$ \\
\hline
\end{tabular}

Table 2. Material parameters

\begin{tabular}{c|c}
\hline $\begin{array}{c}\text { Elasticity modulus of the } \\
\text { primary support } \\
E_{c}(\mathrm{Gpa})\end{array}$ & $\begin{array}{c}\text { Coefficient of the elastic resistance of } \\
\text { surrounding rocks } \\
K(\mathrm{Mpa} / \mathrm{m})\end{array}$ \\
\hline 20 & 400 \\
\hline
\end{tabular}

The contact stress between the shotcrete and the surrounding rocks in the tunnel was measured by TXR-2020 buried stress meters, and displacement was measured by the Leica TS-30 high-precision total station instrument. The measured displacement became stable after 24 days of excavation. The measurement data of the ZK23+590 section at 24 days of excavation are chosen. The contact stresses between the surrounding rocks and the shotcrete as well as displacement at the measuring points are presented in Table 3 and in Table 4. The variation curves of the radial displacements and the contact stress are presented in Fig. 7 and in Fig. 8.

Table 3. Contact stress

\begin{tabular}{c|c|c}
\hline $\begin{array}{c}\text { Number of the Measuring } \\
\text { points }\end{array}$ & $\boldsymbol{\theta}$ Angle $(\mathrm{rad})$ & $\begin{array}{c}\text { Contact stress } \\
p(\mathrm{kPa})\end{array}$ \\
\hline 1 & 0 & 42.3 \\
3 & $\pi / 2$ & 17.8 \\
\hline
\end{tabular}


Table. 4. Vertical and horizontal displacements

\begin{tabular}{c|c|c|c}
\hline $\begin{array}{c}\text { Number of the } \\
\text { Measuring } \\
\text { points }\end{array}$ & $\begin{array}{c}\theta \text { Angle } \\
(\mathrm{rad})\end{array}$ & $\begin{array}{c}\text { Horizontal } \\
\text { displacement } \\
\Delta x(\mathrm{~mm})\end{array}$ & $\begin{array}{c}\text { Vertical } \\
\text { displacement } \\
\Delta y(\mathrm{~mm})\end{array}$ \\
\hline 1 & 0 & 0.2 & 25.1 \\
2 & $\pi / 3$ & 8.7 & 1.7 \\
3 & $\pi / 2$ & 8.3 & 0.3 \\
\hline
\end{tabular}

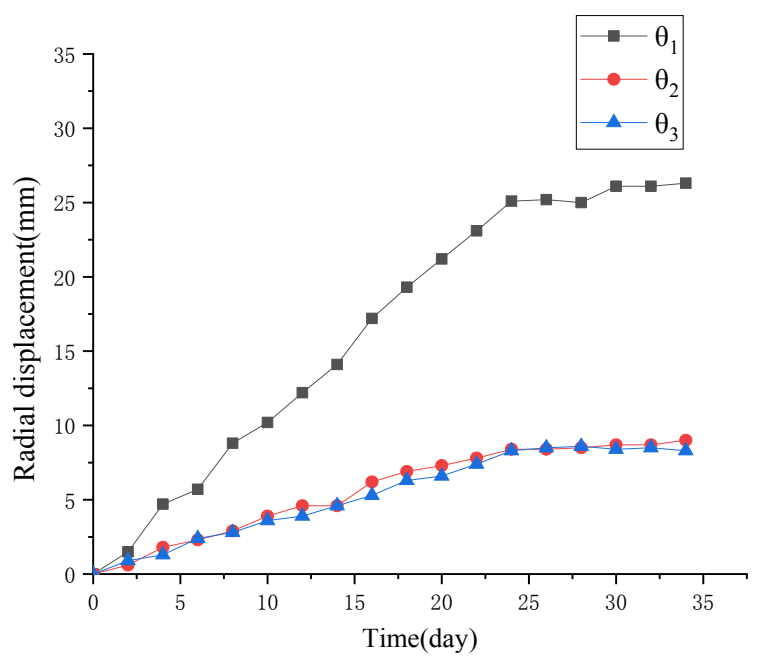

Fig. 7. Variation curve of radial displacement with time

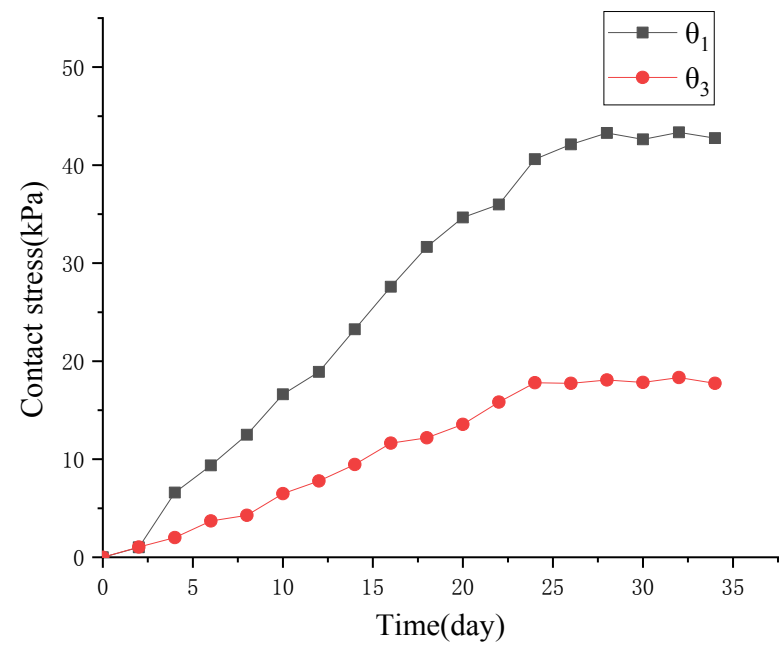

Fig. 8. Variation curve of contact stress with time

\subsection{Internal force analysis of primary support}

Internal forces during the excavation of the tunnel are calculated based on the model, which is constructed in Section 3.3. The sign of measured radial displacement is positive, indicating that the upper bench of the primary support developed free deformation in this stage. Meanwhile, the supporting structure does not bear the elastic resistance. The internal forces of the primary support should be calculated according to Eqs. (27)-(29). The vertical load component and the lateral pressure coefficient at this moment can be calculated by substituting the data in Table 3 into Eqs. (32)-(33). The data in Table 4 are substituted into Eq. (37) so that the integration constants can be calculated. Next, the integration constants and the load parameters $(\bar{p}$ and $\lambda$ ) are brought into the deduced internal force expressions (Eqs. [27]-[29]) to calculate the internal forces.
The internal forces are illustrated in the diagrams (Figs. 911).

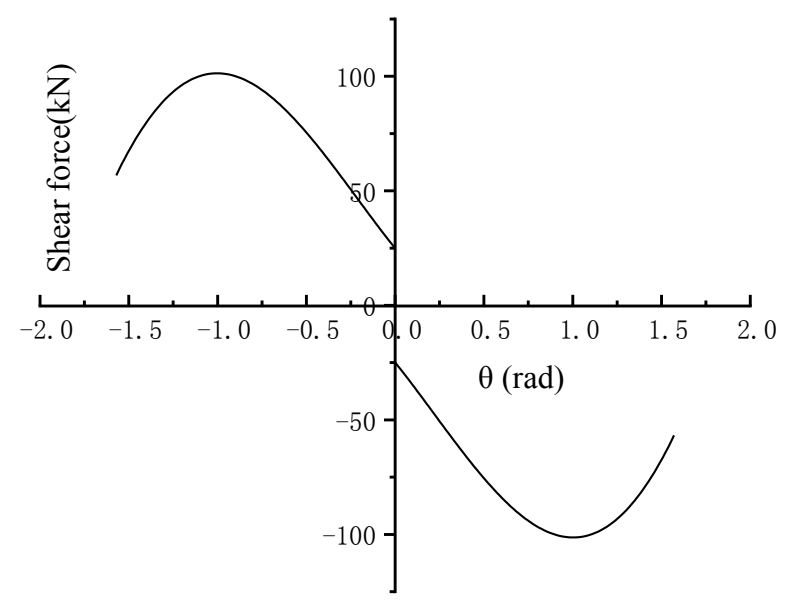

Fig. 9. Shear force of the primary support

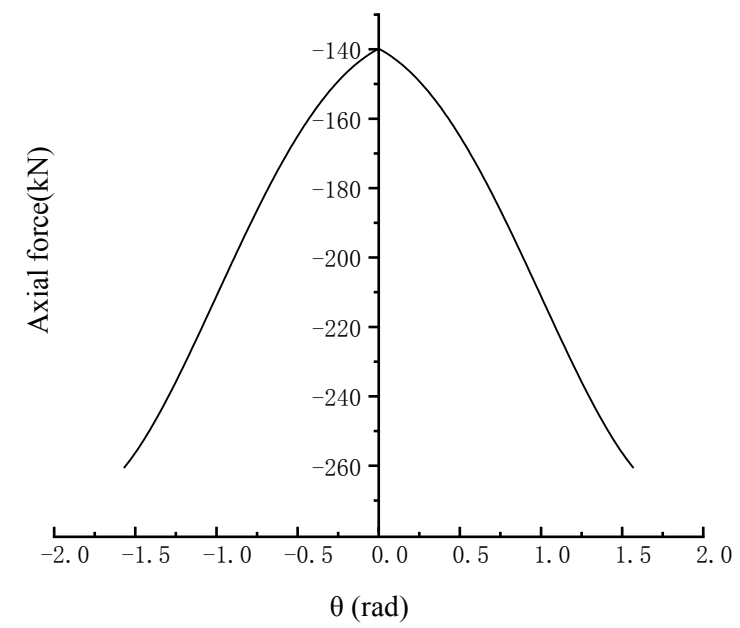

Fig. 10. Axial force of the primary support

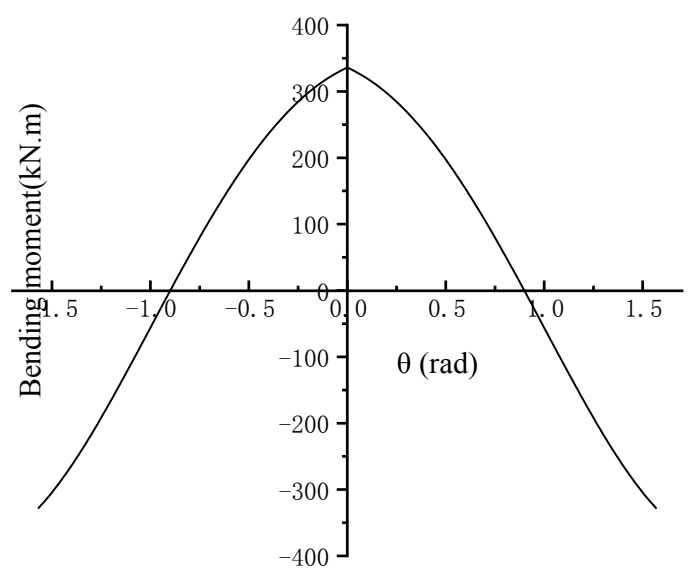

Fig. 11. Bending moment of the primary support

The variation curves of the internal force with time (Figs.12-14) can be obtained by substituting the measurement data in Fig. 7 and in Fig. 8 into Eqs. (27)-(29). 


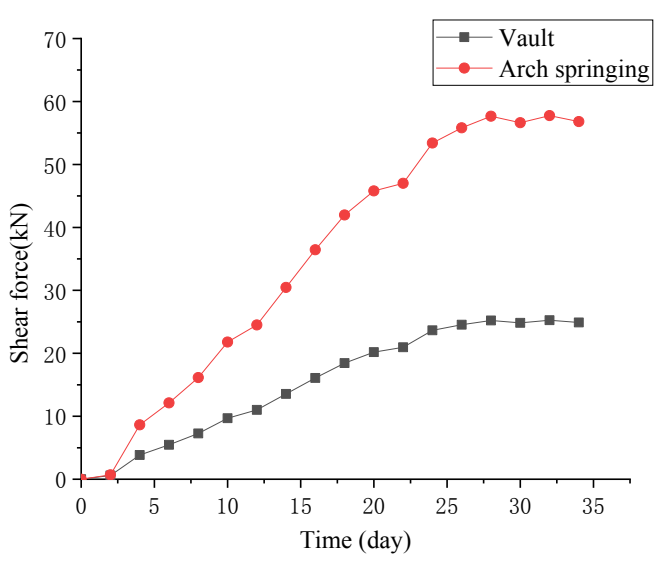

Fig. 12. Variation curve of shear force with time

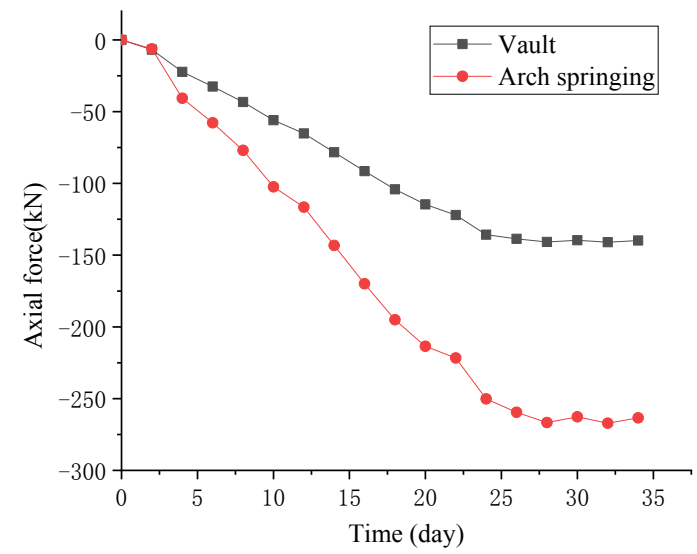

Fig. 13. Variation curve of axial force with time

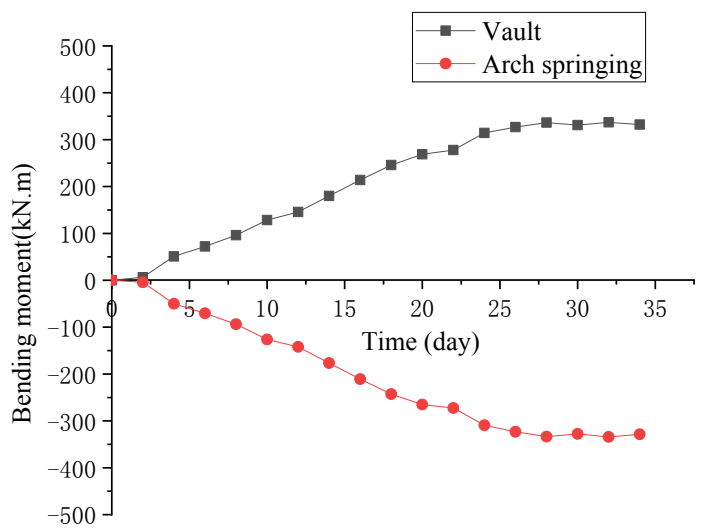

Fig. 14. Variation curve of bending moment with time

It can be observed from Figs.12-14 that with the continuous release of surrounding rock loads, the internal forces of the primary support in the tunnel increases continuously over time and becomes stable after 24 days.

In Figs. 10-12, the shear force of the primary support presents an inverted $\mathrm{V}$-shaped variation with the increase of the $\theta$ angle, reaching the maximum at the position near $60^{\circ}$. The axial force is positively related with the $\theta$ angle and reached the maximum $(260 \mathrm{kN})$ at the arch springing. The bending moment decreased continuously with the increase of the $\theta$ angle. The maximum and the negative bending moments are at the vault and at the arch springing. There is a positive bending moment near the vault in the $\theta$ angle range of about $\pm 45^{\circ}$; however, the bending moment in the rest position is negative. According to internal force distribution characteristics, the arch springing section undertook a large bending moment and axial force, which deserve strong support and monitoring. The material mechanics formula can transform internal force to stress, thus allowing for the assessment of the cracking behavior of the support.

The proposed method is also applicable to the fullsection excavation tunnel. Appropriate displacement measuring points will be added in the process of monitoring. The range of the detachment and the resistance zones could be judged according to the sign of the radial displacement. Later, the internal forces can be calculated utilizing the proposed methods.

\section{Conclusions}

To evaluate the internal forces accurately, a novel backanalysis method based on radial displacement and contact stress was developed to estimate the internal forces of the primary support in deep symmetrically loaded tunnel. A case study was conducted to analyze the mechanical characteristics of the primary support obtained utilizing the proposed methods. The following conclusions could be drawn:

(1) While constructing a symmetrically loaded tunnel, the internal forces of the primary support can be directly evaluated by radial displacement and contact stress.

(2) The axial force is positively correlated with crosssection angle, and the maximum axial force appears near the vault. The shear force presents an inverted $\mathrm{V}$-shape variation with the cross-section angle, and the maximum shear force appears in a $60^{\circ}$ position near the upper arch. The bending moment is negatively correlated with the cross-section angle. The maximum and minimum bending moments appear in the vault and in the arch springing, respectively. The maximum and minimum bending moment values of have opposite signs.

(3) The arch springing is the weak section and should be strengthened by applying a secondary spraying of concrete or by adding a feet-lock bolt. Moreover, the lower benching and the inverted arch should be constructed as soon as possible.

Thus, the back-analysis model based on theory of circular curved beam on elastic foundation was corrected in the proposed method to accurately evaluate the internal forces of the primary support in deep tunnels. However, the proposed method is limited to considering the effect of the symmetrical load. Further study should be conducted to evaluate the internal forces of shallow unsymmetricallyloaded tunnels.

\section{Acknowledgements}

This work was supported by Transportation Science project of Chongqing city (Grant Nos. 2015-03), Science project of Fuling District (Grant Nos. FLKJ2016BBB1082), and the Open Fund Project of Chongqing Jiaotong University Municipal Key Laboratory of Mountain Highway \& Waterway Transportation Geological Disaster Reduction (Grant Nos. kfxm2018-08)

This is an Open Access article distributed under the terms of the Creative Commons Attribution License 


\section{References}

1. Huang, H., Xu, L., "Study on deformation and internal force of surrounding rocks and initial support in Dafengyakou rock road tunnel". Chinese Journal of Rock Mechanics and Engineering, 23(1),2004, pp. 44-52.

2. Scaioni, M., Barazzetti, L., Giussani, A., Previtali, M., Roncoroni, F., Alba, M. I., "Photogrammetric techniques for monitoring tunnel deformation". Earth Science Informatics, 7(2), 2014, pp. 83-95.

3. Ren, S., Tan, Z., Zhang, D., "Research on construction technique of large-section railway tunnels in debris flow strata". China civil engineering journal, 48(S1), 2015, pp. 368-372.

4. Hu, Y., Liu, X., Li, X., Zhang, L., Wang, D., "Engineering risk management of mountain tunnels based on observation". Chinese Journal of Geotechnical Engineering, 32(7), 2010, pp. 1135-1141.

5. Ma, X., Jia, D., Xie, J., "Construction Technology Control of Shallow Buried and Biased Tunnel of Collapsible Loess in High Speed Railway". Journal of Railway Engineering Society, 35(03), 2018, pp. 77-81.

6. Wan, F., Zheng, P., Tan, Z., "Site Monitoring and Analytical research on Mechanical Behaviour of Middle-Wall in DoubleArched Tunnel". Chinese Journal of Underground Space and Engineering, 9(6), 2013, pp. 1355-1361.

7. Gioda, G., Locatelli, L., "Back analysis of the measurements performed during the excavation of a shallow tunnel in sand". International Journal for Numerical and Analytical Methods in Geomechanics, 23(13), 2015, pp. 1407-1425.

8. Miro, S., König, M., Hartmann, D., Schanz, T., “A probabilistic analysis of subsoil parameters uncertainty impacts on tunnelinduced ground movements with a back-analysis study". Computers and Geotechnics, 68, 2015, pp. 38-53.

9. Zhao, C., Lavasan, A. A., Barciaga, T., Zarev, V., Datcheva, M., Schanz, T., "Model validation and calibration via back analysis for mechanized tunnel simulations - The Western Scheldt tunnel case". Computers and Geotechnics, 69(7), 2015, pp. 601-614.

10. Espada, M., Lamas, L., "Back analysis procedure for identification of anisotropic elastic parameters of overcored rock specimens". Rock Mechanics and Rock Engineering, 50(3), 2017, pp. 513-527.

11. Moreira, N., Miranda, T., Pinheiro, M., Fernandes, P., Dias, D., Costa, L., Sena-Cruz, J., "Back analysis of geomechanical parameters in underground works using an Evolution Strategy algorithm". Tunnelling \& Underground Space Technology, 33(1), 2013, pp. 143-158.

12. Dehghan, A. N., Shafiee, S. M., Rezaei, F., "3-D stability analysis and design of the primary support of Karaj metro tunnel: Based on convergence data and back analysis algorithm". Engineering geology, 141, 2012, pp. 141-149.

13. Zhang, Z., Li, X., Li, Y., "Uniqueness of displacement back analysis of a deep tunnel with arbitrary cross section in transversely isotropic rock". International Journal of Rock Mechanics and Mining Sciences, 97, 2017, pp. 110-121.
14. Deng, X., Yuan, D., Yang, D., Zhang, C., "Back Analysis of Geomechanical Parameters of Rock Masses Based on SeepageStress Coupled Analysis". Mathematical Problems in Engineering, 2017(5), 2017, pp. 1-13.

15. Zhu, C., Zhao, H., Zhao, M., "Back analysis of geomechanical parameters in underground engineering using artificial bee colony". The Scientific World Journal, 2014, 2014, pp. 1-13.

16. Miranda, T., Dias, D., Pinheiro, M., Eclaircy-Caudron, S., "Tunnel engineering-influence of the type and the quantity of measurements in the back analysis of geomechanical parameters". European Journal of Environmental and Civil Engineering, 20(1), 2016, pp. 60-78.

17. Miranda, T., Dias, D., Eclaircy-Caudron, S., Correia, A. G., Costa, L., "Back analysis of geomechanical parameters by optimisation of a 3D model of an underground structure". Tunnelling and Underground Space Technology, 26(6), 2011, pp. 659-673.

18. Yazdani, M., Sharifzadeh, M., Kamrani, K., Ghorbani, M., "Displacement-based numerical back analysis for estimation of rock mass parameters in Siah Bisheh powerhouse cavern using continuum and discontinuum approach". Tunnelling and Underground Space Technology incorporating Trenchless Technology Research, 28(1), 2012, pp. 41-48.

19. Kodama, J., Miyamoto, T., Kawasaki, S., Fujii, Y., Kaneko, K., Hagan, P., "Estimation of regional stress state and Young's modulus by back analysis of mining-induced deformation". International Journal of Rock Mechanics and Mining Sciences, 63, 2013, pp. 1-11.

20. Janin, J. P., Dias, D., Emeriault, F., Kastner, R., Le Bissonnais, H., Guilloux, A., "Numerical back-analysis of the southern Toulon tunnel measurements: a comparison of 3D and 2D approaches". Engineering Geology, 195, 2015, pp. 42-52.

21. Janin, J. P., "Tunnels en milieu urbain: Prévisions des tassements avec prise en compte des effets des pré-soutènements (renforcement du front de taille et voûte-parapluie)". Doctoral Dissertation of INSA de Lyon, France, 2012. pp. 141-152.

22. Sun, J., Qi, Y., "Normal calculation-back analysis of surrounding rock stability of subsee tunnel". Rock and Soil Mechanics, 31(8), 2010, pp. 2353-2360.

23. Wen, J., Zhang, Y., Wang, C., "Back analysis of internal force of initial support in tunnel based on touch stress". Rock and Soil Mechanics, 32(8),2011, pp. 2467-2472.

24. Wen, J., Zhang, Y., Wang, C., Jiang, Z., "Back analysis for the mechanical properties of initial tunnel support based on steel arch stresses". China civil engineering journal, 45(2),2012, pp. 170-175.

25. Wang, X., Shao, Z., "Theoretical Research on the Inverse Analysis of the Internal Force of the Primary Support in a Horseshoe Tunnel". Modern Tunnelling Technology, 51(6),2014, pp. 83-87.

26. Cheng, Y., Cui, G., Song, L., "Theoretical study on inversion analysis of internal force of support structure in multi-circle arc tunnel”. Modern Tunnelling Technology, 55(1),2018, pp. 85-89. 